\title{
PERFORMANCE ANALYSIS OF COMPOSITE SEMICIRCULAR BREAKWATERS OF DIFFERENT CONFIGURATIONS AND POROSITIES
}

\author{
Hee Min Teh ${ }^{1}$, Vengatesan Venugopal ${ }^{2}$ and Tom Bruce $^{3}$
}

\begin{abstract}
The perforated free surface semicircular breakwater developed by Teh et al. (2010) was experimentally proven to be an effective anti-reflection structure with high energy dissipation ability. However, the performance characteristics of the breakwater deteriorated with a decrease in the immersion depth and an increase in wavelength. To enhance the performance of the breakwater with limited immersion depth, wave screens of different configurations and porosities were introduced below the free surface semicircular caisson. The hydrodynamic characteristics of these composite breakwaters were investigated in irregular waves using physical modelling. Comparisons of the experimental results showed that the semicircular caisson with a double screen of $25 \%$ porosity was a better breakwater configuration compared to that with a single screen. The extension of wave screen was also found to be particularly helpful in attenuating longer waves.
\end{abstract}

Keywords: free surface breakwater, semicircular breakwater, wave screen, wave transmission, wave reflection, energy dissipation, horizontal wave forces.

\section{INTRODUCTION}

Free surface breakwaters, also known as open breakwaters, have generated a great deal of interest in the coastal and ocean engineering in recent years. They are essentially barriers located near the free surface where the energy flux is greatest. Such barriers do not extend over the whole water depth, permitting water circulation beneath the structure. These breakwaters could be installed on a group of piles. These structures control the height of the waves mainly by reflection and energy loss, and have been found to be most effective when used at locations where wave conditions are relatively mild.

Teh et al. (2010) developed a perforated free surface semicircular breakwater (SCB) that was particularly suitable to be used in near-shore waters. Over the tested range, the SCB was an effective energy dissipater and a reasonable wave reflector. The experimental results indicated that the wave attenuation performance of the SCB model was somewhat less satisfactory at lower immersion depths, particularly when subjected to longer period waves, due to substantial transmission of waves underneath the structure. The wave transmission for the structure at lower immersion ranged from about $60 \%-98 \%$, which is rather high for many coastal and marine related applications. Hence the aim of the present study is to reduce the transmission level by introducing wave screens that are directly attached underneath the SCB model. The hydrodynamic characteristics of the combined semicircular breakwater with screens of different configurations and porosities are investigated under irregular waves in a wave flume. The main purpose of the experimental exercise is to evaluate the effectiveness of such a composite breakwater in lower immersion depth.

The present study aims to address the aforementioned problem by introducing a wave screen(s) of various porosities underneath the SCB. The hydrodynamic characteristics of the test models of different configurations and porosities are investigated using physical modelling approach. This experimental exercise is to improve the design of the SCB so as to give satisfactory performance when the structure is subjected to limited immersion.

\section{BACKGROUND LITERATURE}

There are various designs of free surface breakwaters developed to provide wave protection to small ports and marinas. Teh et al. (2010) classified the fixed free surface breakwater designs based on their

\footnotetext{
${ }^{1}$ Lecturer, Civil Engineering Department, Universiti Teknologi PETRONAS, Bandar Seri Iskandar, Perak, Malaysia.

${ }^{2}$ Lecturer, Institute for Energy Systems, School of Engineering, University of Edinburgh, The King's Buildings, Edinburgh, UK.

${ }^{3}$ Senior lecturer, Institute for Energy Systems, School of Engineering, University of Edinburgh, The King's Buildings, Edinburgh, UK.
} 


\section{COASTAL ENGINEERING 2012}

configurations into four categories: solid-type, caisson-type, plate-type and multipart-type. The solid-type barriers, which are generally simple in design and have high effective mass for stability, reduce the wave energy mainly reflection. Caisson-type barriers are quite similar to the solid-type in terms of their physical appearance but these are with interference chambers in the structures for further energy dissipation. Platetype barriers consist of a single or a combination of multiple plates with different alignments located at various submergence depths in the water domain; whilst the multipart-type barriers are made of a large number of structural elements (e.g. pipes, concrete and wooden planks, vertical rods, etc) and present in complex forms in the sea domain. The multipart-type barriers are highly porous to the incoming waves, thus reducing the amount of horizontal wave force and reflection in front of the structures.

Wave screens have a number of desirable features that have encouraged their use within harbours, i.e. easy navigation within the harbour due to reduced wave activity, permission of water exchange and maintenance of water quality within the basin, and reduced wave loads on the barrier. The basic structure of a wave screen consists of a series of slots or holes so that energy is dissipated in the viscous eddy formed by the flow through the perforations. They do reflect wave energy from the screen face and the intensity mainly depends on the porosity and configuration of the screen. In general, there are two types of wave screens used in harbour, namely (a) the horizontally slotted screens and (b) the closely spaced piles.

\section{(a) Horizontally slotted screens}

A typical slotted screen is composed of a series of closely spaced elements (e.g. precast concrete or timber planks) mounted on a supporting frame extending from the seabed to well above the water surface. For a single screen with low porosity, wave reflection is less influenced by the change of wave height because there is little flow through the screen and most of the energy is reflected. The influence of screen porosity on wave reflection is only apparent when the wave heights are small (Bennett et al., 1992). In some cases, a solid back wall or a similar perforated screen is placed at a distance away from the front screen to enhance the wave tranquillity level in within the harbours; however, standing waves may formed within the space. Allsop and Hettiarachchi (1988) studied the screens of $14 \%-28 \%$ porosities with respect to a broad range of relative screen spacing, $0<B / L<1.2$. They found that the lowest wave reflection occurred at $B / L \approx 0.25$ and 0.75 , and the highest reflections at $B / L \approx 0.5$ and 1.0 ; and the influence of screen porosity was only apparent when the wave reflection was small. McBride (1994) proposed several simple design formulae to predict the reflection performance of single and double wave screens. The slotted screens can also be formed by a series of horizontally placed circular elements, in which the details were discussed by Balaji and Sundar (2002) and Krishnakumar et al. (2010).

\section{(b) Pile breakwaters}

A pile breakwater is typically constructed by a row or multiple rows of closely spaced piles extending from the seabed to some distance over the water surface. In practice, construction of these breakwaters is much too difficult and expensive to drive single piles closely together (Allsop, 1995). Nonetheless, these breakwaters are still commonly used as wave barriers in many ports and harbours. The performance of these barriers mainly depends on the geometry and size of the piles, the pile spacing and their distribution. The study of wave interaction on the barriers with rectangular piles was studied by Huang (2007), Heikal et al. (2007) and Koraim (2007); whilst those with circular piles was investigated by Subba Rao et al. (1999), Yagci et al. (2006), Koraim (2007) and Heikal et al. (2007). A comparison of results by Koraim (2007) showed that the square pile breakwater was more efficient than the circular piles in wave attenuation by $5 \%$ $-15 \%$, and the efficiency increased with a decrease in pile spacing and an increase in the span width.

\section{EXPERIMENTAL SETUP}

In the present study, a semicircular breakwater model with a front wall porosity of 9\% (SCB9), as shown in Figure 1, was selected. The semicircular caisson was made of a semi-cylindrical PVC tube with a wall thickness of $10 \mathrm{~mm}$. The front wall perforation of SCB9, which contained 6 rows by 4 columns of $10 \mathrm{~mm} \mathrm{x}$ $60 \mathrm{~mm}$ rectangular openings spread out across the quadrant surface area, were arranged to produce various levels of energy dissipation during the passage of water flow. To reduce the overtopping discharge, the rear wall was perforated by 2 rows by 4 columns of $30 \mathrm{~mm} \times 60 \mathrm{~mm}$ rectangular openings close to the crown. The radius and breakwater length perpendicular to the wave direction for the model were $0.25 \mathrm{~m}$ and 0.395 m, respectively. Further details of the test model were presented by Teh et al. $(2011 ; 2012)$. 


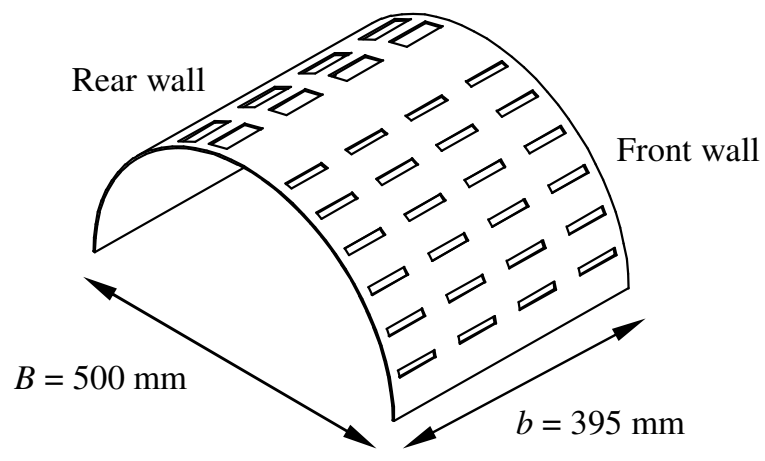

Figure 1: SCB9 model

A 300 -mm screen of various porosities (i.e. $\mathcal{E}=25 \%, 40 \%$ and $50 \%$ ) was extended vertically from the bottom of the front or/and rear wall(s) of the SCB9 caisson. Three configurations of wave screen were tested, i.e. a front screen (FS), a rear screen (RS) and a double screen (DS). Each wave screen consisted of a number of closely-spaced rectangular metal plates, each of $39.5 \mathrm{~mm}$ long, $30 \mathrm{~mm}$ wide and $10 \mathrm{~mm}$ thick. There were four holes drilled through the centre of the plate so that the plate could be fixed to a vertical connecting bar by means of screws. The $280 \mathrm{~mm}$-long connecting bar had a square cross section of $40 \mathrm{~mm}$ x $40 \mathrm{~mm}$. There were 27 pairs of 5-mm circular holes with full penetration through the bar, evenly distributed along the full length of the bar with a space interval of $10 \mathrm{~mm}$. The matrix of the circular holes on the bar allowed attachment of horizontal plates of various spacing between them, which in turn led to variation of the wave screen's porosity. The gap between SCB9 and the screen was $5 \mathrm{~mm}$. The properties of the wave screen of varying porosities are displayed in Table 1.

Table 1: Properties of the wave screen(s)

\begin{tabular}{ccc}
\hline $\begin{array}{c}\text { Porosity of wave } \\
\text { screen }\end{array}$ & Number of plates & $\begin{array}{c}\text { Spacing between plates } \\
(\mathrm{mm})\end{array}$ \\
\hline $25 \%$ & 7 & 10 \\
$40 \%$ & 6 & 20 \\
$50 \%$ & 5 & 30 \\
\hline
\end{tabular}

To install the front screen in the wave flume, the screen component was firmly fixed to an end of a watertight S-type loadcell and the other end of the loadcell was rigidly fastened to a suspended ' $L$ ' shape mounting frame that was securely fixed to the tops of the wave flume. The vertical length of the frame was adjustable to enable the variation of the submergence of the wave screen. The sharp edges of the frame were trimmed and the thickness of the frame perpendicular to the wave advance was kept to $20 \mathrm{~mm}$ so that the presence of the frame would not pose significant interference to the flow. The width of the frame was designed at $100 \mathrm{~mm}$ to resist flexure caused by the maximum horizontal wave force acting on the wave screen. Figure 2 illustrates the complete set-up of a wave screen in water. The installation principles for the rear screen are similar to those of the front screen. Instead, the 'I' shape mounting frame was used to hold the rear screen in place. It is important to note that the wave screens tested in this study were detached from the free surface barrier (i.e. SCB9) and the side walls so as to ensure the entire horizontal wave forces were transferred to the load cell for measurement. In the present experimental study, considering the limitations of the wave flume width no attempt has been made to model the pile supporting system. 


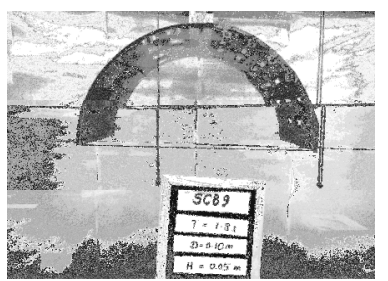

(a) SCB9

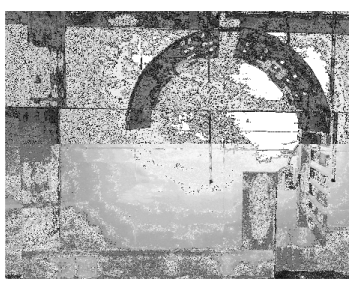

(b) SCB9 with front screen of $40 \%$ porosity (SCB9-FS40)

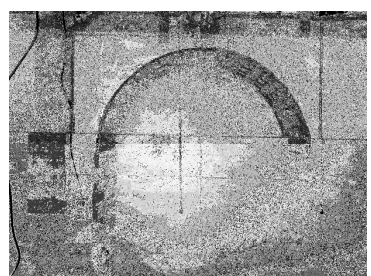

(c) SCB9 with rear screen of $50 \%$ porosity (SCB9-RS50)

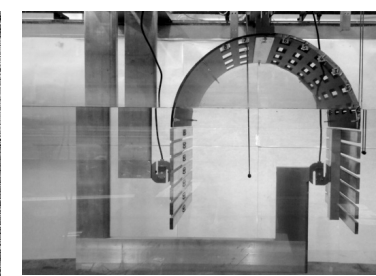

(d) SCB9 with double screens of $25 \%$ porosity (SCB9-DS25)

Figure 2: Test models for SCB9 and wave screen(s)

The laboratory tests were conducted in a $22 \mathrm{~m}$ long, $0.4 \mathrm{~m}$ wide and $0.7 \mathrm{~m}$ deep wave flume in the Hydraulics Laboratory of the School of Engineering, the University of Edinburgh. A schematic diagram of the experimental setup is shown in Figure 3. The flap-type, active absorption wave generator developed by Edinburgh Designs was used to produce both regular and irregular waves. At the down-wave end of the flume, passive wave absorbing "beach", which was made of two pieces of triangular upright foam, were installed to minimise the reflection of incident waves from the end wall of the wave flume during the experiments. The test section was located at a distance of $12 \mathrm{~m}$ from the wave generator. Six resistance type wave probes (WP1 - WP6) were used to record the water surface elevations at different locations in the flume. The probes WP1, WP2 and WP3 located offshore of the model were used to separate the incident and reflected waves using the Least Square method developed by Mansard and Funke (1980). The wave probe WP3 was located seaward of the structure with a distance of at least half of the longest wave length generated in the flume. The separations between WP1, WP2 and WP3 were altered for each peak wave period. The probe WP4 located at a distance of $50 \mathrm{~mm}$ from the seaward wall of the model was used to measure the surface elevation just in front of the model. For the SCB9 model, probe WP5 was positioned through one of the rectangular openings near the crown to measure the fluctuation of water level within the breakwater's chamber. The transmitted waves were measured by probe WP6, which was located at a distance $2.5 \mathrm{~m}$ from the leeward wall of the model. The horizontal wave forces acting on the SCB model were measured by two load cells (LC1 and LC2) attached at both ends of the model at the crest through a specially designed mounting frame, in which the details are described by Teh et al. (2011, 2012). For the wave screens, the total horizontal wave force on the respective structures was measured by S-type load cells of IP68 supplied by Ningbo Xinlan Electric Appliances Co. Ltd, China. These equipments were carefully calibrated before serious measurements.

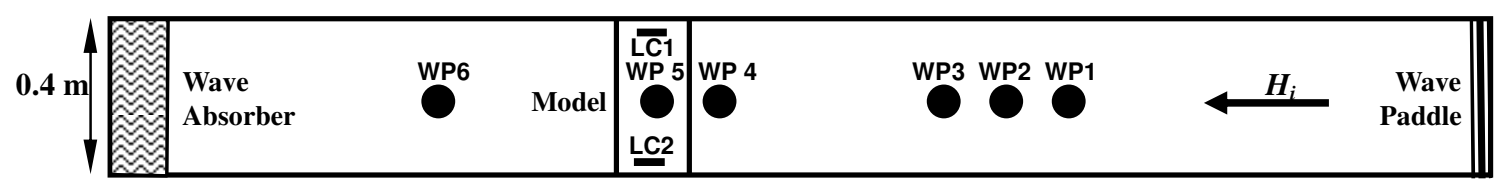

PLAN VIEW

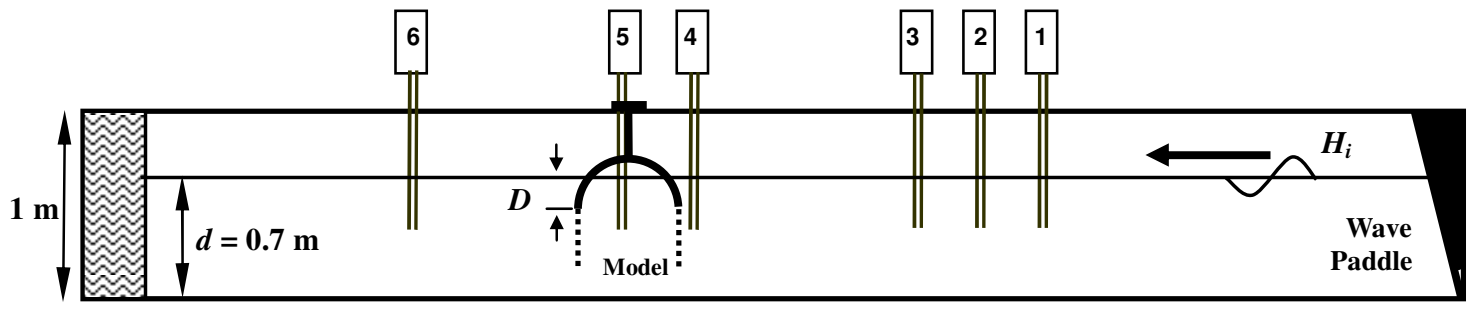

SIDE VIEW

Figure 3: Laboratory set-up 
A series of experiments were conducted in both regular and irregular wave conditions at a water depth of $0.7 \mathrm{~m}$. The experimental results for only irregular waves are reported in this paper. In all irregular wave tests, waves were generated according to JONSWAP spectrum with a peak enhancement factor of 3.3, using the software 'Ocean and Wave' developed by the Edinburgh Designs, UK . The test models were subjected to 12 peak periods, $T_{p}$ ranging from $0.7-1.8 \mathrm{~s}$ at intervals of $0.1 \mathrm{~s}$. For each peak period, at least four different significant wave heights, $H_{m 0}$, ranging from $0.04-0.14 \mathrm{~m}$ were used. This yielded a range of wave steepness, $H_{m 0} / L_{p}$ varying from $0.01-0.10$ (where $L_{p}$ is the wavelength corresponding to the peak period). Data acquisition and analysis were carried out by using Aalborg University's WAVELAB TM software. The sampling duration for each run was $256 \mathrm{~s}$, with a sampling frequency of $100 \mathrm{~Hz}$.

In this study, an emphasis was given to the SCB9 immersed in limited depth of which it performed inadequately. An immersion of $50 \mathrm{~mm}$ from the still water level was selected to give the ratio $D / d=0.071$, where $D$ and $d$ are the breakwater draft and water depth respectively. The test environments encompassed both deep and intermediate water conditions. In total, approximately 380 test runs were conducted.

\section{DISCUSSION OF RESULTS}

Hydraulic performance of the test models are evaluated in terms of wave transmission, wave reflection, energy dissipation, and wave climate in the proximity; whereas, the peak horizontal wave forces under both wave crests and troughs acting on the respective test models are also assessed accordingly.

\section{Wave Transmission}

The transmission coefficient, $C_{T}$, which is the ratio of the transmitted wave height-to-the incident wave height, often used to reflect the wave attenuation ability of a breakwater, e.g. a lower $C_{T}$ value indicates the breakwater is an effective wave attenuator. The plots (a) - (c) in Figure 4 present the transmission coefficients for three breakwater configurations, i.e. with a front screen (SCB9-FS), a rear screen (SCB9RS) and a double screen (SCB9-DS), and they are plotted with respect to the relative breakwater width, $B / L_{p}$. For each breakwater configuration, three screen porosities were tested, i.e. $\varepsilon=25 \%, 40 \%$ and $50 \%$. It is apparent from the plots that the $C_{T}$ of the test models decreases with an increase in $B / L_{p}$. For a particular breakwater configuration, it is found that wave screen with smaller porosity gives higher wave attenuation ability. The influence of screen porosity is more profound for the case of the double screens (SCB9-DS) models. A comparison between the breakwater configurations shows that the front screen (SCB9-FS) models offer the least suppression to waves; whilst the SCB9-DS models provide the greatest performance with attenuation of more than $50 \%$ of the incident wave heights at $B / L_{p}>0.4$. It is also noticed that the SCB9 model with double screens of $25 \%$ porosity (SCB9-DS25) outperforms the other test models with greater porosities, bringing down the $C_{T}$ variation to $0.29-0.76$ for the tested range of $B / L_{p}$.

\section{Wave Reflection}

Wave reflection characteristics of the test models are represented by the reflection coefficient, $C_{R}$, which is the ratio of the reflected wave height-to-the incident wave height, e.g. a lower $C_{R}$ value implies the breakwater is an effective anti-reflection structure. The $C_{R}$ of the test models are demonstrated in plots (d) (f) in Figure 4. It is seen from these plots that the $C_{R}$ values increase as $B / L_{p}$ increases or the screen porosity decreases. The $C_{R}$ recorded range from $0.13-0.44,0.13-0.41$ and $0.14-0.46$ for the SCB9-FS, SCB9RS and SCB9-DS models, respectively. The amount of reflection demonstrated by the test models are relatively small compared to those posed by the vertical wall breakwaters. For a given $B / L_{p}$, the variations of $C_{R}$ with the change of screen porosity are barely more than 0.20 regardless of the breakwater configurations. The SCB9 with a double screen display higher reflection characteristics than the SCB9 with

a single screen; while the SCB9-RS models are slightly less reflective than the SCB9-FS models due to increased energy dispersion within the interference chamber of the SCB9-RS models. 

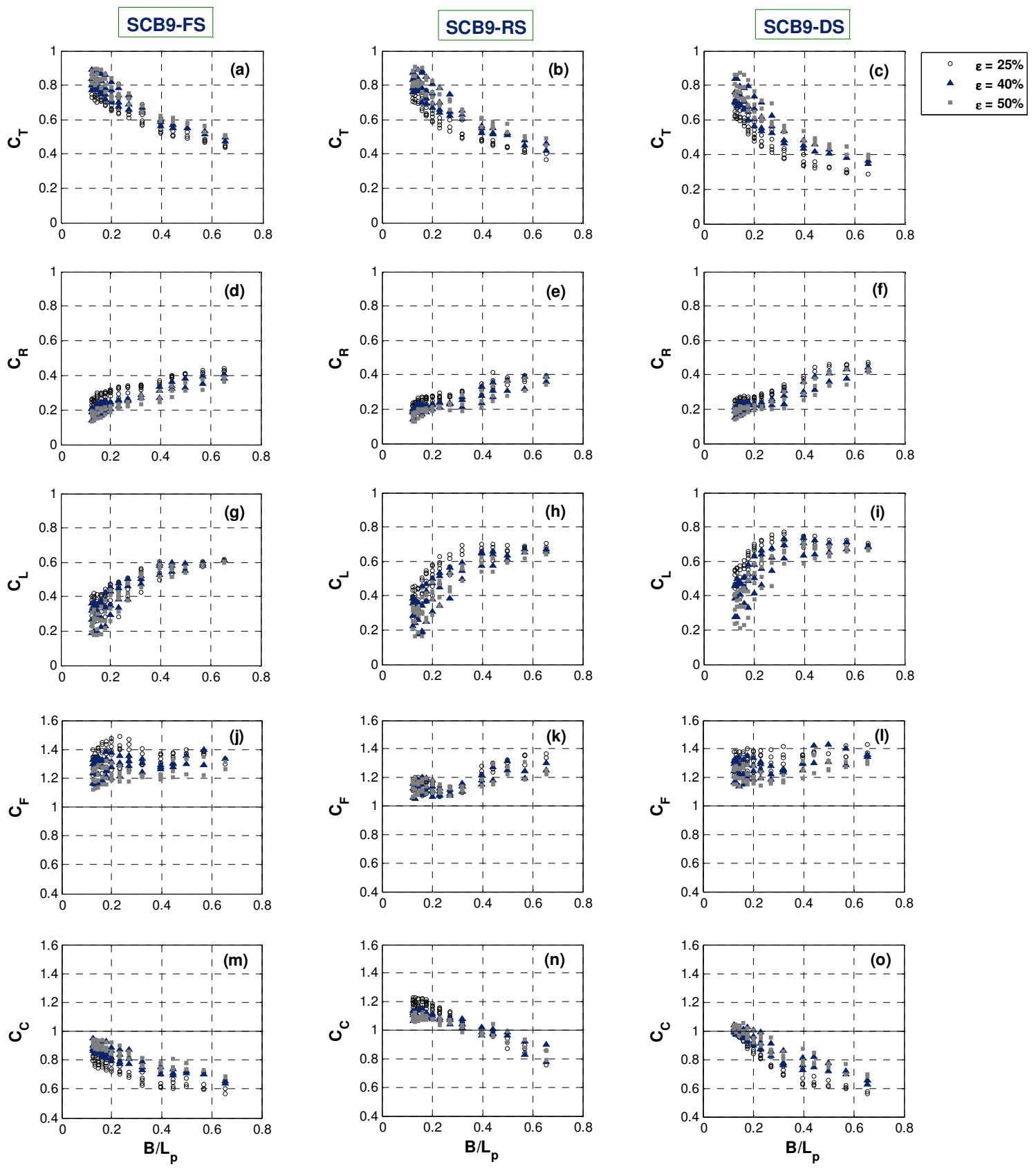

Figure 4: Hydraulic performance of the test models

\section{Energy Loss}

Since the energy dissipated at the breakwater involves complicated processes and is difficult to measure experimentally, it is therefore mathematically estimated based on the principle of conservation of energy, giving the energy dissipation coefficient, $C_{L}=1-C_{T}{ }^{2}-C_{R}{ }^{2}$. The $C_{L}$ value indicates the percentage of the energy dissipated at the breakwater by the incident waves. Hence, a good energy dissipater always yields a high $C_{L}$ value. The plots (g) - (i) in Figure 4 show the energy dissipation characteristics of the respective breakwater models. All the plots demonstrate a drastic increase of $C_{L}$ as $B / L_{p}$ increases to 0.4 for the SCB9FS and SCB9-RS models, and 0.3 for the SCB9-DS models; and the $C_{L}$ seem to retain at a constant value 
thereafter without much variations. The plots also reveal that the $C_{L}$ increases with a decrease of the screen porosity irrespective of the breakwater configurations. From the experimental results, it can be learnt that the test models are highly dissipative, particularly when exposed to shorter period waves.

\section{Wave Climate in Front of the Breakwater}

Wave climate in front of the test models is characterised by a wave disturbance coefficient, $C_{F}$, which is a ratio of the wave height right in front of the breakwater-to-the incident wave height. The $C_{F}$ values of the test models are presented in plots (j) - (1) in Figure 4. The $C_{F}$ values of all the test models are constantly more than unity, showing an amplification of wave height in front of the breakwaters. The wave behaviours in front of the SCB9-FS and SCB9-DS models are almost alike, in which the $C_{F}$ gradually vary between 1.2 and 1.4 within the tested range of $B / L_{p}$; whilst the $C_{F}$ of the SCB9-RS models are comparatively small. The influence of screen porosity is found to be dominant, i.e. the smaller the porosity, the stronger the wave agitation in front of the breakwaters. It is also realised that the variations of $C_{F}$ for the test models seem to be in proportion with their respective $C_{R}$ as seen in plots (d) - (f), indicating that the wave climate in front of the breakwaters is primarily governed by the effect of wave reflection by the structures.

\section{Wave Climate in the Breakwater's Chamber}

Wave climate in the breakwater chamber is characterised by another wave disturbance coefficient, $C_{C}$, which is a ratio of the wave height within the breakwater-to-the incident wave height. The $C_{C}$ of the SCB9FS, SCB9-RS and SCB9-DS models are shown in plots (m) - (o) in Figure 4. The plots show a decrease of $C_{C}$ with an increase in $B / L_{p}$. Both SCB9-FS and SCB9-DS models exhibit similar wave climate in their chambers for a given screen porosity and wave condition; the larger the screen porosity, the greater will be the $C_{C}$ values for the breakwater models. A comparison between the $C_{T}$ in plot (a) and the $C_{C}$ in plot (m) shows that the $C_{T}$ is smaller than the $C_{C}$ by the order of 0.2 for the SCB9-FS models. Similar comparison is made for the SCB9-DS models in plots (c) and (o) and the results demonstrate a difference of approximately 0.3 between $C_{T}$ and $C_{C}$. These variations suggest the efficiency of the rear elements, i.e. the rear wall of the SCB9 and the wave screen, in wave attenuation. On the other hand, the wave climate in the chambers of the SCB9-RS models strongly depends on the relative breakwater width. For $B / L_{p}<0.4$, breakwater with a rear screen of higher porosity tends to induce lesser $C_{C}$ values; and for $B / L_{p}>0.4$, the influence of screen porosity on $C_{C}$ is rather weak.

\section{Horizontal Wave Loadings}

Figure 5 demonstrates the total horizontal wave forces (i.e. a sum of the forces by the SCB9 and the screen(s)) on the composite models corresponding to the significant wave height, $H_{s}$. The forces are characterised by the average of the highest one-third of the force data, $F_{1 / 3}$, in which a positive value indicates the force by wave crests (positive force), and a negative value indicates the force by wave troughs (negative force). From the figure, a linear relation can be found between $F_{1 / 3}$ and $H_{s}$ for all the test models. The $F_{1 / 3}$ does not seem to be affected by the change of wave periods; however, the influence of screen porosity on $F_{1 / 3}$ is fairly noticeable from the figure, particularly for negative forces. As expected, the increment of wave forces is subjected to the increase of the total frontal area of the screen in the direction of the wave propagation, i.e. $F_{1 / 3}$ increases with the decreasing screen porosity. The positive forces of the models are always greater than the negative ones, and the variations are particularly marked for the SCB9FS models. In term of the force response to the incident wave heights, the positive forces of the SCB9-RS and SCB9-DS models are somewhat similar and happen to be more significant than the SCB9-FS models. The increased intensity of the positive force response of the former is mainly attributed to wave interception by the solid rear wall of the SCB9; and that of the latter is entirely due to energy transfer to the double screens. Likewise, higher wave response is also observed for the SCB9-DS models when subjected to wave troughs. Whereas, the negative wave responses of the SCB9-FS model are comparable to those of the SCB9-RS models. 
(a) SCB9-FS

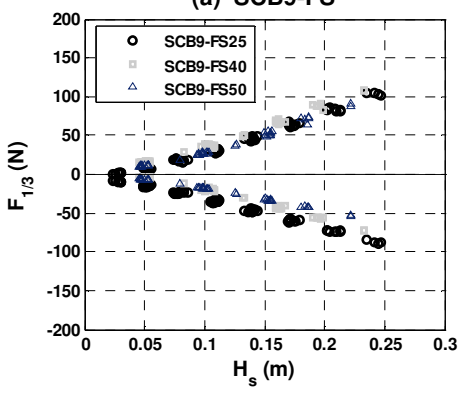

(b) SCB9-RS

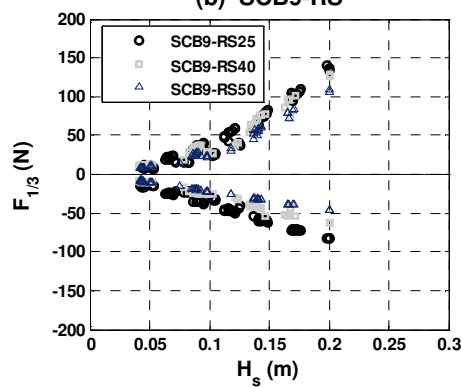

(c) SCB9-DS

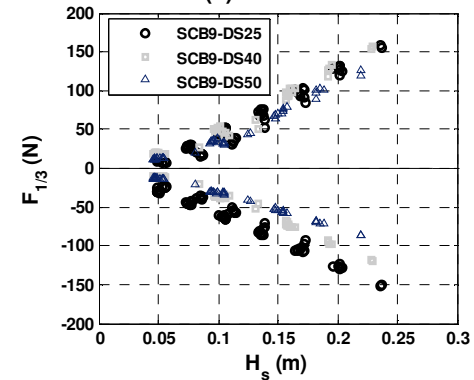

Figure 5: Total horizontal wave forces for test models

Figure 5 gives an overview of the total horizontal wave forces acting on the respective test models; however, it does not reflect the distribution of the wave forces. Figure 6 presents the horizontal wave forces measured on the respective elements of the test models, i.e. the SCB9 and wave screen. The forces acting on the SCB9 models, $F_{\mathrm{SCB} 9}{ }^{+}$and $F_{\mathrm{SCB} 9}{ }^{-}$are less sensitive to the change of the screen porosity, especially at smaller wave heights. Near the free surface, the increase of $F_{\mathrm{SCB} 9}{ }^{+}$is far more rapid than that of $F_{\mathrm{SCB} 9}{ }^{-}$for a given incident wave height. The previous findings by Goda (1995) also exhibit the similar characteristics for a vertical wall, where maximum positive force and almost zero negative force would be anticipated at free surface. On the contrary, for the wave screens that are fully submerged and located near the mid-depth the horizontal forces by wave trough $\left(F_{\mathrm{FS}}{ }^{-}\right)$are found to be higher than those by wave crest $\left(F_{\mathrm{FS}}{ }^{+}\right)$for a given wave height. This behaviour was also observed by Goda (1995) of which the peak negative force occurs at a short distance above the mid-depth for a vertical wall. Apart from this, the same tendency was also reported by McConnell et al. (1999) for vertical structures, and Wang (2006) for the bottom seated semicircular breakwater in the presence of pulsating waves. Thus, for the wave screens built in relatively deep water, there is a possibility that the horizontal wave forces acting under a wave trough becomes a critical design factor rather than the wave forces under a wave crest.

For the SCB9-DS models, the loading behaviours and the forces imposed on the SCB9 and the front screens have a close correspondence to those of the SCB9-FS models as shown in Figure 6 (a) - (c). The forces on the rear screens, $F_{\mathrm{RS}}{ }^{+}$and $F_{\mathrm{RS}}{ }^{-}$are relatively small due to reduced wave activity in the breakwaters' chamber as presented in Figure 4(o). For the SCB9-RS models, a drastic increase of the $F_{\mathrm{SCB} 9}{ }^{+}$ is seen for all the test models in Figure 6 (d) - (f) due to wave interception by the solid rear wall of the SCB9.

\section{Optimum Breakwater Design}

A review of the previous discussions of the experimental results shows that the SCB9 model with double screens of $25 \%$ porosity (SCB9-DS25) could be the most optimum breakwater design due to its high hydraulic efficiency. The combination of the structural elements makes it highly resistive to waves and it is capable of dampening up to $70 \%$ of the incident wave heights. The energy dissipation characteristics of the breakwater are far more advanced than its reflection ability, i.e. the maximum energy loss induced by the breakwater is about 3 times the energy reflected from the structure. Therefore, the SCB9-DS25 is regarded as an effective energy dissipater with minimal reflection effect. 

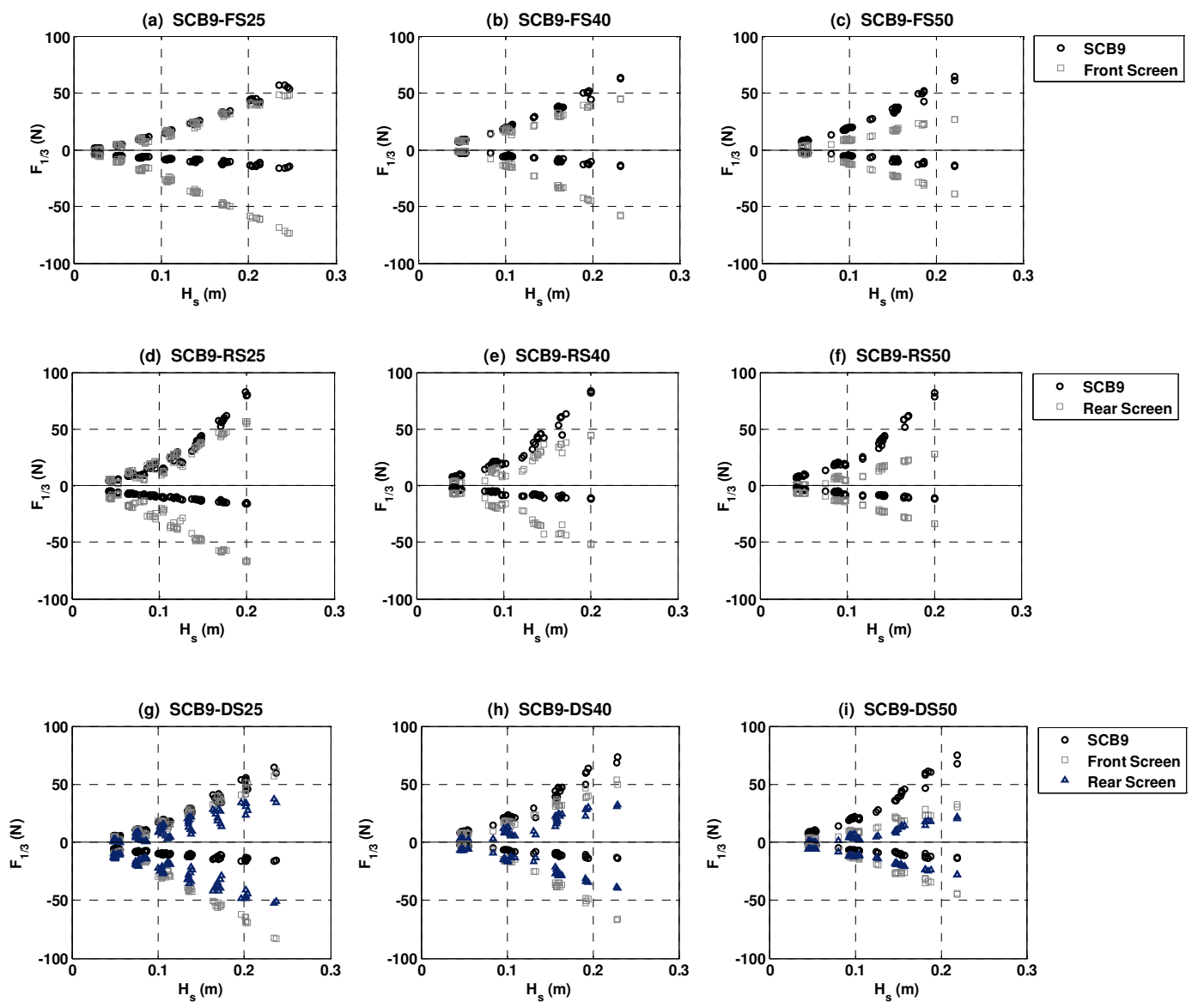

Figure 6: Measured horizontal wave force on the respective elements of the test models

\section{CONCLUSIONS}

A free surface semicircular breakwater model with a front wall porosity of 9\% (SCB9) coupled with an extension of a front screen (SCB9-FS), a rear screen (SCB9-RS) or a double screen (SCB9-DS) from its bottom, was tested in a wave flume under irregular waves of different significant wave heights and peak wave periods. The screen porosities selected for the tests were $25 \%, 40 \%$ and $50 \%$. The hydraulic characteristics of the test models of different configurations and porosities were evaluated by estimating the coefficients of transmission $\left(C_{T}\right)$, reflection $\left(C_{R}\right)$ and energy dissipation $\left(C_{L}\right)$, as well as the wave climate coefficients in front of the breakwater $\left(C_{F}\right)$ and in the breakwater's chamber $\left(C_{C}\right)$. The horizontal wave loadings on the models were also measured and reported. The following conclusions were reached within the limitations of the test programme:

- The SCB9-DS models offered a better wave attenuation performance (with more than 50\% suppression of the incident wave heights at $B / L_{p}>0.4$ ) compared to the single-screened breakwaters.

- The composite breakwaters were effective anti-reflection structures with energy reflected from the structures was consistently less than $25 \%$.

- The SCB9-DS models were highly dissipative with a rate of dissipation up to $78 \%$ of the incident wave energy.

- For the composite breakwaters tested in the experiments, the maximum wave heights in front of the breakwater was about 1.5 times the incident wave heights; whilst the maximum wave height in the chambers was approximately 1.25 times the incident wave heights. 
- The performance enhancement due to the wave screen was proven to be significant in terms of wave attenuation and energy dissipation; and the resulting increment of reflection characteristics was comparatively small.

- The total horizontal wave forces on the SCB9-DS models were higher than those of the SCB9-FS and SCB9-RS models by an order of approximately 1.6 due to the additional forces imposed to the rear screen.

- The SCB9-DS models with a screen porosity of $25 \%$ was proposed to be the most optimum breakwater design that provides good wave protection, effective energy dissipation and minimal wave reflection when immersed in limited depth.

\section{ACKNOWLEDGMENTS}

We acknowledge the financial support by Institute for Energy Systems of School of Engineering, the University of Edinburgh. We would also like to thank Universiti Teknologi PETRONAS (Malaysia) for providing scholarship for this study.

\section{REFERENCES}

Allsop, N.W.H. 1995. Vertical walls and breakwaters: Optimisation to improve vessel safety and wave disturbance by reducing wave reflections. In: Wave Forces on Inclined and Vertical Wall Structures, ASCE, New York. 232 -258.

Allsop, N. W., and Hettiarachchi, S. S. 1988. Reflections from Coastal Structures. Proceedings of the $21^{\text {st }}$ International Coastal Engineering Conference, vol. 1, 782-794.

Balaji, R. and Sundar, V. 2002. Hydraulic performance of double screen breakwaters. The $5^{\text {th }}$ International Conference on Hydro-Science \& Engineering (ICHE-2002), Warsaw, Poland, September 18-21.

Bennett, G. S., McIver, P. and Smallman, J. V. 1992. A mathematical model of a slotted wavescreen breakwater. Journal of Coastal Engineering 18, 231-249.

Goda, Y. 1995. Japan's design practice in assessing wave forces on vertical breakwaters. In: Wave Forces on Inclined and Vertical Wall Structures, ASCE, New York. 140 -155.

Heikal, E. M., Salem, T. N. and Koraim, A. S. 2007. Theoretical study for one row of piles used as a breakwater. Mansoura Engineering Journal, March 2007, Egypt.

Huang, Z. H. 2007. Wave interaction with one or two rows of closely spaced rectangular cylinders. Journal of Ocean Engineering 34, 1584-1591.

Koraim, A. S. 2007. Efficiency of double rows of piles used as a breakwater. Mansora Engineering Journal, March 2007, Egypt.

Krishnakumar, C., Sundar, V. and Sannasiraj, S. A. 2010. Hydrodynamic performance of single- and double-wave screens. Journal of Waterway, Port, Coastal and Ocean Engineering, Jan/Feb 2010, 59-65.

Mansard, E.P.D. and Funke, E.R. 1980. The mesurement of incident and reflected spectra using a least squares method. Proceedings of the $17^{\text {th }}$ Coastal Engineering Conference, 154-172.

McBride, M. W., Smallman, J. V. and Allsop, N. W. H. 1994. Numerical model of absorbing wave screens. Report IT400, HR Wallingford, October 1994.

McConnell, K. J., Allsop, N. W. H. and Flohr, H. 1999. Seaward wave loading on vertical coastal structures. Proceedings of the International Conference on Coastal Structure '99, Vol. 1, Santander, Spain.

Subba Rao, Rao, N.B.S. and Sathyanarayana 1999. Laboratory investigation on wave transmission through two rows of perforated hollow piles. Ocean Engineering 26, 675-699.

Teh, H. M., Venugopal, V. and Bruce, T. 2010. Hydrodynamic performance of a free surface semicircular perforated breakwater. Proceedings of the $32^{\text {nd }}$ International Conference on Coastal Engineering, Shanghai, China.

Teh, H. M., Venugopal, V. and Bruce, T. 2011. Performance analysis of a semicircular free surface breakwater. Proceedings of the $30^{\text {th }}$ International Conference on Ocean, Offshore and Arctic Engineering, Rotterdam, The Netherlands.

Teh, H. M., Venugopal, V. and Bruce, T. 2012. Hydrodynamic characteristics of a free surface semicircular breakwater exposed to irregular waves, Journal of Waterway, Port, Coastal and Ocean Engineering, vol. $138(2), 149-163$. 
Wang, L. Q. 2006. The study of 2-d and 3-d random waves acting on a semicircular breakwater. PhD thesis, Dalian University of Technology, China.

Yagci, O., Kirca, V. S. O., Kabdasli, M. S., Celik, A. O., Unal, N. E. and Aydingakko, A. 2006. An experimental model application of wavescreen: Dynamic pressure, water particle velocity, and wave measurements. Ocean Engineering 33, 1299-1321. 